\title{
ON MONOIDS OF MONOTONE INJECTIVE PARTIAL SELFMAPS OF INTEGERS WITH COFINITE DOMAINS AND IMAGES
}

\author{
OLEG GUTIK AND DUŠAN REPOVŠ
}

\begin{abstract}
We study the semigroup $\mathscr{I}_{\infty}^{\nearrow}(\mathbb{Z})$ of monotone injective partial selfmaps of the set of integers having cofinite domain and image. We show that $\mathscr{I}_{\infty}^{\nearrow}(\mathbb{Z})$ is bisimple and all of its non-trivial semigroup homomorphisms are either isomorphisms or group homomorphisms. We also prove that every Baire topology $\tau$ on $\mathscr{I}_{\infty}^{\nearrow}(\mathbb{Z})$ such that $\left(\mathscr{I}_{\infty}^{\nearrow}(\mathbb{Z}), \tau\right)$ is a Hausdorff semitopological semigroup is discrete and we construct a non-discrete Hausdorff inverse semigroup topology $\tau_{W}$ on $\mathscr{I}_{\infty}^{\nearrow}(\mathbb{Z})$. We show that the discrete semigroup $\mathscr{I}_{\infty}^{\nearrow}(\mathbb{Z})$ cannot be embedded into some classes of compact-like topological semigroups and that its remainder under the closure in a topological semigroup $S$ is an ideal in $S$.
\end{abstract}

\section{INTRODUCTION AND PRELIMINARIES}

In this paper all spaces will be assumed to be Hausdorff. We shall denote the first infinite cardinal by $\omega$ and the cardinality of the set $A$ by $|A|$. Also, we shall denote the additive group of integers by $\mathbb{Z}(+)$. We shall identify all sets $X$ with their cardinality $|X|$.

For a topological space $X$, a family $\left\{A_{s} \mid s \in \mathscr{A}\right\}$ of subsets of $X$ is called locally finite if for every point $x \in X$ there exists an open neighbourhood $U$ of $x$ in $X$ such that the set $\left\{s \in \mathscr{A} \mid U \cap A_{s} \neq \varnothing\right\}$ is finite. A subset $A$ of $X$ is said to be

- co-dense in $X$ if $X \backslash A$ is dense in $X$;

- an $F_{\sigma}$-set in $X$ if $A$ is an intersection of a countable family of open subsets in $X$.

We recall that a topological space $X$ is said to be

- compact if each open cover of $X$ has a finite subcover;

- countably compact if each open countable cover of $X$ has a finite subcover;

- pseudocompact if each locally finite open cover of $X$ is finite;

- a Baire space if for each sequence $A_{1}, A_{2}, \ldots, A_{i}, \ldots$ of nowhere dense subsets of $X$ the union $\infty$ $\bigcup A_{i}$ is a co-dense subset of $X$;

$i=1$

- Čech complete if $X$ is Tychonoff and for every compactification $c X$ of $X$ the remainder $c X \backslash X$ is an $F_{\sigma}$-set in $c X$;

- locally compact if every point of $X$ has an open neighbourhood with a compact closure.

According to Theorem 3.10.22 of [14], a Tychonoff topological space $X$ is pseudocompact if and only if each continuous real-valued function on $X$ is bounded.

An algebraic semigroup $S$ is called inverse if for any element $x \in S$ there exists a unique $x^{-1} \in S$ such that $x x^{-1} x=x$ and $x^{-1} x x^{-1}=x^{-1}$. The element $x^{-1}$ is called the inverse of $x \in S$. If $S$ is an inverse semigroup, then the function inv $S \rightarrow S$ which assigns to every element $x$ of $S$ its inverse element $x^{-1}$ is called an inversion.

A band is a semigroup of idempotents. If $S$ is a semigroup, then we shall denote the subset of idempotents in $S$ by $E(S)$. If $S$ is an inverse semigroup, then $E(S)$ is closed under multiplication. The semigroup operation on $S$ determines the following partial order $\leqslant$ on $E(S): e \leqslant f$ if and only if ef $=f e=e$. This order is called the natural partial order on $E(S)$. A semilattice is a commutative semigroup of idempotents. A semilattice $E$ is called linearly ordered or a chain if its natural order is

Date: November 7, 2018.

2010 Mathematics Subject Classification. Primary 22A15, 20M20. Secondary 20M18, 54D40, 54E52, 54H10, 54H15.

Key words and phrases. Topological semigroup, semitopological semigroup, semigroup of bijective partial transformations, symmetric inverse semigroup, ideal, homomorphism, Baire space, semigroup topologization, bicyclic semigroup. 
a linear order. A maximal chain of a semilattice $E$ is a chain which is properly contained in no other chain of $E$.

If $h: S \rightarrow T$ is a homomorphism from a semigroup $S$ into a semigroup $T$ then we say that $h$ is:

- a trivial homomorphism if $h$ is either an isomorphism or an annihilating homomorphism;

- a group homomorphism if $(S) h$ is a subgroup of $T$.

If $\mathfrak{C}$ is an arbitrary congruence on a semigroup $S$, then we denote by $\Phi_{\mathfrak{C}}: S \rightarrow S / \mathfrak{C}$ the natural homomorphism from $S$ onto the quotient semigroup $S / \mathfrak{C}$. A congruence $\mathfrak{C}$ on a semigroup $S$ is called non-trivial if $\mathfrak{C}$ is distinct from universal and identity congruences on $S$, and a group congruence if the quotient semigroup $S / \mathfrak{C}$ is a group. Every inverse semigroup $S$ admits a least (minimal) group congruence $\mathfrak{C}_{m g}$ :

$$
a \mathfrak{C}_{m g} b \text { if and only if there exists } e \in E(S) \text { such that } a e=b e
$$

(see Lemma III.5.2 of [26]).

The Axiom of Choice implies the existence of maximal chains in any partially ordered set. According to [26], a chain $L$ is called an $\omega$-chain if $L$ is isomorphic to $\{0,-1,-2,-3, \ldots\}$ with the usual order $\leqslant$. Let $E$ be a semilattice and $e \in E$. We denote $\downarrow e=\{f \in E \mid f \leqslant e\}$ and $\uparrow e=\{f \in E \mid e \leqslant f\}$. By $\left(\mathscr{P}_{<\omega}(\lambda), \subseteq\right)$ we shall denote the free semilattice with identity over a set of cardinality $\lambda \geqslant \omega$, i.e., $\left(\mathscr{P}_{<\omega}(\lambda), \subseteq\right)$ is the set of all finite subsets (including the empty set) of $\lambda$ with the semilattice operation "union".

If $S$ is a semigroup, then we shall denote the Green relations on $S$ by $\mathscr{R}, \mathscr{L}, \mathscr{J}, \mathscr{D}$ and $\mathscr{H}$ (see Section 2.1 of [9]):

$$
\begin{aligned}
& a \mathscr{R} b \text { if and only if } a S^{1}=b S^{1} ; \\
& a \mathscr{L} b \text { if and only if } S^{1} a=S^{1} b ; \\
& a \mathscr{J} b \text { if and only if } S^{1} a S^{1}=S^{1} b S^{1} ; \\
& \mathscr{D}=\mathscr{L} \circ \mathscr{R}=\mathscr{R} \circ \mathscr{L} ; \\
& \mathscr{H}=\mathscr{L} \cap \mathscr{R} .
\end{aligned}
$$

A semigroup $S$ is called simple if $S$ does not contain any proper two-sided ideals and bisimple if $S$ has only one $\mathscr{D}$-class.

A semitopological (resp. topological) semigroup is a Hausdorff topological space together with a separately (resp. jointly) continuous semigroup operation. An inverse topological semigroup with the continuous inversion is called a topological inverse semigroup. A Hausdorff topology $\tau$ on a (inverse) semigroup $S$ such that $(S, \tau)$ is a topological (inverse) semigroup is called a (inverse) semigroup topology.

If $\alpha: X \rightarrow Y$ is a partial map, then by $\operatorname{dom} \alpha$ and $\operatorname{ran} \alpha$ we shall denote the domain and the range of $\alpha$, respectively. Let $\mathscr{I}_{\lambda}$ denote the set of all partial injective transformations of an infinite set $X$ of cardinality $\lambda$ together with the following semigroup operation: $x(\alpha \beta)=(x \alpha) \beta$ if $x \in \operatorname{dom}(\alpha \beta)=\{y \in$ $\operatorname{dom} \alpha \mid y \alpha \in \operatorname{dom} \beta\}$, for $\alpha, \beta \in \mathscr{I}_{\lambda}$. The semigroup $\mathscr{I}_{\lambda}$ is called the symmetric inverse semigroup over the set $X$ (see Section 1.9 of [9]). The symmetric inverse semigroup was introduced by Wagner [29] and it plays a major role in the theory of semigroups.

Let $\mathbb{Z}$ be the set of integers with the usual order $\leqslant$. We shall say that a partial map $\alpha: \mathbb{Z} \rightarrow \mathbb{Z}$ is monotone if $n \leqslant m$ implies $(n) \alpha \leqslant(m) \alpha$ for $n, m \in \mathbb{Z}$. By $\mathscr{I}_{\infty}^{\nearrow}(\mathbb{Z})$ we denote a subsemigroup of injective partial monotone selfmaps of $\mathbb{Z}$ with cofinite domains and images, i.e.,

$$
\mathscr{I}_{\infty}^{\nearrow}(\mathbb{Z})=\left\{\alpha \in \mathscr{I}_{\omega} \mid \alpha \text { is monotone, }|\mathbb{Z} \backslash \operatorname{dom} \alpha|<\infty \quad \text { and } \quad|\mathbb{Z} \backslash \operatorname{ran} \alpha|<\infty\right\}
$$

Obviously, $\mathscr{I}_{\infty}^{\nearrow}(\mathbb{Z})$ is an inverse submonoid of the semigroup $\mathscr{I}_{\omega}$. We observe that $\mathscr{I}_{\infty}^{\nearrow}(\mathbb{Z})$ is a countable semigroup. Furthermore, we shall denote the identity of the semigroup $\mathscr{I}_{\infty}^{\nearrow}(\mathbb{Z})$ by $\mathbb{I}$ and the group of units of $\mathscr{I}_{\infty}^{\nearrow}(\mathbb{Z})$ by $H(\mathbb{I})$. 
Lemma 1.1. A partial injective monotone map $\alpha$ is an element of the semigroup $\mathscr{I}_{\infty}^{\nearrow}(\mathbb{Z})$ if and only if there exist integers $d_{\alpha}$ and $u_{\alpha}$ such that the following conditions hold:

$$
(m-1) \alpha=(m) \alpha-1 \text { and }(n+1) \alpha=(n) \alpha+1 \quad \text { for all integers } m \leqslant d_{\alpha} \text { and } n \geqslant u_{\alpha},
$$

and $\alpha \in H(\mathbb{I})$ if and only if $(n+1) \alpha=(n) \alpha+1$ for any integer $n$.

Proof. The implication $(\Leftarrow)$ is trivial.

$(\Rightarrow)$ Since for every element $\alpha$ of the semigroup $\mathscr{I}_{\infty}^{\nearrow}(\mathbb{Z})$ the sets $\mathbb{Z} \backslash \operatorname{dom} \alpha$ and $\mathbb{Z} \backslash$ ran $\alpha$ are finite, we conclude that there exist integers $d_{\alpha}$ and $u_{\alpha}$ such that the following condition holds:

$$
m, n \in \operatorname{dom} \alpha \cap \operatorname{ran} \alpha, \quad \text { for all } m \leqslant d_{\alpha} \text { and } n \geqslant u_{\alpha} \text {. }
$$

Now, since the partial map $\alpha: \mathbb{Z} \rightarrow \mathbb{Z}$ is monotone we have that

$$
(m-1) \alpha \leqslant(m) \alpha-1 \text { and }(n) \alpha+1 \leqslant(n+1) \alpha \text { for all } m \leqslant d_{\alpha} \text { and } n \geqslant u_{\alpha},
$$

and hence we get that

$$
\begin{aligned}
& (m-j) \alpha \leqslant(m-(j-1)) \alpha-1 \leqslant \cdots \leqslant(m) \alpha-j \quad \text { and } \\
& (n) \alpha+j \leqslant \cdots \leqslant(n+j-1) \alpha+1 \leqslant(n+j) \alpha
\end{aligned}
$$

for any positive integer $j, m \leqslant d_{\alpha}$ and $n \geqslant u_{\alpha}$. Then by condition (1) we have that

$$
(m-1) \alpha=(m) \alpha-1 \text { and }(n+1) \alpha=(n) \alpha+1 \quad \text { for all integers } m \leqslant d_{\alpha} \text { and } n \geqslant u_{\alpha} .
$$

It is obvious that if $(n+1) \alpha=(n) \alpha+1$ for any integer $n$ then $\alpha: \mathbb{Z} \rightarrow \mathbb{Z}$ is a bijective monotone map and hence $\alpha \in H(\mathbb{I})$. Conversely, if $\alpha \in H(\mathbb{I})$ then $\alpha: \mathbb{Z} \rightarrow \mathbb{Z}$ is a bijective monotone map and the first assertion of lemma implies that $(n+1) \alpha=(n) \alpha+1$ for any integer $n$.

The bicyclic semigroup $\mathscr{C}(p, q)$ is the semigroup with the identity 1 generated by elements $p$ and $q$ subject only to the condition $p q=1$. The bicyclic semigroup is bisimple and every one of its congruences is either trivial or a group congruence. Moreover, every non-annihilating homomorphism $h$ of the bicyclic semigroup is either an isomorphism or the image of $\mathscr{C}(p, q)$ under $h$ is a cyclic group (see Corollary 1.32 in [9]).

The bicyclic semigroup plays an important role in the algebraic theory of semigroups and in the theory of topological semigroups. For example, the well-known result of Andersen [1] states that a $(0-)$ simple semigroup is completely $(0-)$ simple if and only if it does not contain the bicyclic semigroup.

Remark 1.2. Let $n$ be an arbitrary integer. We put $\mathscr{C}(n,+)$ and $\mathscr{C}(n,-)$ to be semigroups which are generated by partial transformations $\alpha_{n}^{+}$and $\beta_{n}^{+} ; \alpha_{n}^{-}$and $\beta_{n}^{-}$, respectively, of the set of integers $\mathbb{Z}$, defined as follows:

$$
\begin{array}{ll}
(i) \alpha_{n}^{+}=\left\{\begin{array}{cl}
i, & \text { if } i \leqslant n ; \\
i+1, & \text { if } i>n,
\end{array}\right. & (i) \beta_{n}^{+}=\left\{\begin{array}{cl}
i, & \text { if } i \leqslant n ; \\
i-1, & \text { if } i>n+1,
\end{array}\right. \\
(i) \alpha_{n}^{-}=\left\{\begin{array}{cl}
i, & \text { if } i \geqslant n ; \\
i-1, & \text { if } i<n,
\end{array}\right. & (i) \beta_{n}^{-}=\left\{\begin{array}{cl}
i, & \text { if } i \geqslant n ; \\
i+1, & \text { if } i<n-1,
\end{array}\right.
\end{array}
$$

$i \in \mathbb{Z}$. We remark that $\mathscr{C}(n,+)$ and $\mathscr{C}(n,-)$ are bicyclic semigroups for every positive integer $n$. Therefore the semigroup $\mathscr{I}_{\infty}^{\nearrow}(\mathbb{Z})$ contains infinitely many isomorphic copies of the bicyclic semigroup $\mathscr{C}(p, q)$.

We shall say that a partial map $\alpha: \mathbb{Z} \rightarrow \mathbb{Z}$ is almost monotone if there exists a finite subset $F$ in $\operatorname{dom} \alpha$ such that the restriction $\left.\alpha\right|_{\operatorname{dom} \alpha \backslash F}: \mathbb{Z} \rightarrow \mathbb{Z}$ is a monotone partial map. By $\mathscr{I}_{\infty}^{\rightarrow}(\mathbb{Z})$ we denote a subsemigroup of injective partial almost monotone selfmaps of $\mathbb{Z}$ with cofinite domains and images, i.e.,

$$
\mathscr{I}_{\infty}^{\not \rightarrow}(\mathbb{Z})=\left\{\alpha \in \mathscr{I}_{\omega} \mid \alpha \text { is almost monotone, }|\mathbb{Z} \backslash \operatorname{dom} \alpha|<\infty \quad \text { and } \quad|\mathbb{Z} \backslash \operatorname{ran} \alpha|<\infty\right\} .
$$

Obviously, $\mathscr{I}_{\infty}^{\rightarrow}(\mathbb{Z})$ is an inverse submonoid of the semigroup $\mathscr{I}_{\omega}$ and $\mathscr{I}_{\infty}^{\nearrow}(\mathbb{Z})$ is an inverse submonoid of $\mathscr{I}_{\infty}^{\rightarrow}(\mathbb{Z})$. We observe that $\mathscr{I}_{\infty}^{\rightarrow}(\mathbb{Z})$ is a countable semigroup. 
It is well known that topological algebra studies the influence of topological properties of its objects on their algebraic properties and the influence of algebraic properties of its objects on their topological properties. There are two main problems in topological algebra: the problem of non-discrete topologization and the problem of embedding into objects with some topological-algebraic properties.

In mathematical literature the question about non-discrete (Hausdorff) topologization was posed by Markov [23]. Pontryagin gave well known conditions for a base at the unity of a group for its non-discrete topologization (see Theorem 4.5 of [19] or Theorem 3.9 of [27]). Various authors have refined Markov's question: can a given infinite group $G$ endowed with a non-discrete group topology be embedded into a compact topological group? Again, for an arbitrary Abelian group $G$ the answer is affirmative, but there is a non-Abelian topological group that cannot be embedded into any compact topological group (see Section 9 of [10]).

Also, Ol'shanskiy [25] constructed an infinite countable group $G$ such that every Hausdorff group topology on $G$ is discrete. Eberhart and Selden [13] showed that every Hausdorff semigroup topology on the bicyclic semigroup $\mathscr{C}(p, q)$ is discrete. Bertman and West [7] proved that every Hausdorff topology $\tau$ on $\mathscr{C}(p, q)$ such that $(\mathscr{C}(p, q), \tau)$ is a semitopological semigroup is also discrete. Taimanov [28] gave sufficient conditions on a commutative semigroup to have a non-discrete semigroup topology.

Many mathematiciants have studied the problems of embeddings of topological semigroups into compact or compact-like topological semigroups (see [8]). Neither stable nor $\Gamma$-compact topological semigroups can contain a copy of the bicyclic semigroup [3, 20]. Also, the bicyclic semigroup cannot be embedded into any countably compact topological inverse semigroup [17]. Moreover, the conditions were given in [5] and [6] when a countably compact or pseudocompact topological semigroup cannot contain the bicyclic semigroup.

However, Banakh, Dimitrova and Gutik [6] have constructed (assuming the Continuum Hypothesis or the Martin Axiom) an example of a Tychonoff countably compact topological semigroup which contains the bicyclic semigroup. The problems of topologization of semigroups of partial transformations and their embeddings into compact-like semigroup were studied in [15, 16].

We showed in [18] that the semigroup $\mathscr{I}_{\infty}^{\nearrow}(\mathbb{N})$ of partial cofinite monotone injective transformations of the set of positive integers $\mathbb{N}$ has algebraic properties similar to those of the bicyclic semigroup: it is bisimple and all of its non-trivial semigroup homomorphisms are either isomorphisms or group homomorphisms. We proved that every locally compact topology $\tau$ on $\mathscr{I}_{\infty}^{\nearrow}(\mathbb{N})$ such that $\left(\mathscr{I}_{\infty}^{\nearrow}(\mathbb{N}), \tau\right)$ is a topological inverse semigroup, is discrete and we described the closure of $\left(\mathscr{I}_{\infty}^{\nearrow}(\mathbb{N}), \tau\right)$ in a topological semigroup.

In this paper we shall describe Green relations on $\mathscr{I}_{\infty}^{\nearrow}(\mathbb{Z})$, show that $\mathscr{I}_{\infty}^{\nearrow}(\mathbb{Z})$ is bisimple and all of its non-trivial semigroup homomorphisms are either isomorphisms or group homomorphisms. We shall also prove that every Baire topology $\tau$ on $\mathscr{I}_{\infty}^{\nearrow}(\mathbb{Z})$ such that $\left(\mathscr{I}_{\infty}^{\nearrow}(\mathbb{Z}), \tau\right)$ is a Hausdorff semitopological semigroup is discrete and construct a non-discrete Hausdorff semigroup inverse topology $\tau_{W}$ on $\mathscr{I}_{\infty}^{\nearrow}(\mathbb{Z})$. We shall show that the discrete semigroup $\mathscr{I}_{\infty}^{\nearrow}(\mathbb{Z})$ cannot be embedded into some classes of compactlike topological semigroups and that its remainder under the closure in a topological semigroup $S$ is an ideal in $S$.

\section{Algebraic properties of the Semigroup $\mathscr{I}_{\infty}^{\nearrow}(\mathbb{Z})$}

Proposition 2.1. (i) An element $\alpha$ of the semigroup $\mathscr{I}_{\infty}^{\nearrow}(\mathbb{Z})$ is an idempotent if and only if $(x) \alpha=x$ for every $x \in \operatorname{dom} \alpha$.

(ii) If $\varepsilon, \iota \in E\left(\mathscr{I}_{\infty}^{\nearrow}(\mathbb{Z})\right)$, then $\varepsilon \leqslant \iota$ if and only if $\operatorname{dom} \varepsilon \subseteq \operatorname{dom} \iota$.

(iii) The semilattice $E\left(\mathscr{I}_{\infty}^{\nearrow}(\mathbb{Z})\right)$ is isomorphic to $\left(\mathscr{P}_{<\omega}(\mathbb{Z}), \subseteq\right)$ under the mapping $(\varepsilon) h=\mathbb{Z} \backslash$ dom $\varepsilon$.

(iv) Every maximal chain in $E\left(\mathscr{I}_{\infty}^{\nearrow}(\mathbb{Z})\right)$ is an $\omega$-chain.

(v) $\alpha \mathscr{R} \beta$ in $\mathscr{I}_{\infty}^{\nearrow}(\mathbb{Z})$ if and only if $\operatorname{dom} \alpha=\operatorname{dom} \beta$.

(vi) $\alpha \mathscr{L} \beta$ in $\mathscr{I}_{\infty}^{\nearrow}(\mathbb{Z})$ if and only if $\operatorname{ran} \alpha=\operatorname{ran} \beta$.

(vii) $\alpha \mathscr{H} \beta$ in $\mathscr{I}_{\infty}^{\nearrow}(\mathbb{Z})$ if and only if $\operatorname{dom} \alpha=\operatorname{dom} \beta$ and $\operatorname{ran} \alpha=\operatorname{ran} \beta$.

(viii) $\mathscr{I}_{\infty}^{\nearrow}(\mathbb{Z})$ is a simple semigroup and hence $\mathscr{J}=\mathscr{I}_{\infty}^{\nearrow}(\mathbb{Z}) \times \mathscr{I}_{\infty}^{\nearrow}(\mathbb{Z})$. 
(ix) For all idempotents $\varepsilon, \varphi \in \mathscr{I}_{\infty}^{\nearrow}(\mathbb{Z})$ there exist infinitely many elements $\alpha, \beta \in \mathscr{I} \Phi_{\infty}(\mathbb{Z})$ such that $\alpha \cdot \beta=\varepsilon$ and $\beta \cdot \alpha=\varphi$.

Proof. Statements $(i)-(i v)$ are trivial and they follow from the definition of the semigroup $\mathscr{I}_{\infty}^{\nearrow}(\mathbb{Z})$.

The proofs of $(v)-(v i i)$ follow trivially from the fact that $\mathscr{I}_{\infty}^{\nearrow}(\mathbb{Z})$ is a regular semigroup, and Proposition 2.4.2 and Exercise 5.11.2 in 21.

(viii) Note that every cofinite subset of $\mathbb{Z}$ is order-isomorphic to $\mathbb{Z}$. Let $\varphi, \gamma \in \mathscr{I}_{\infty}^{\nearrow}(\mathbb{Z})$ be arbitrary. Since the $\operatorname{sets} \mathbb{Z} \backslash \operatorname{dom} \varphi, \mathbb{Z} \backslash \operatorname{dom} \gamma$ and $\mathbb{Z} \backslash \operatorname{ran} \varphi$ are finite and the $\operatorname{sets} \operatorname{dom} \varphi, \operatorname{dom} \gamma$ and $\operatorname{ran} \varphi$ are order-isomorphic to $\mathbb{Z}$, we conclude that there exist bijective monotone maps $\varphi_{\text {dom }}: \operatorname{dom} \varphi \rightarrow \mathbb{Z}$, $\gamma_{\text {dom }}: \operatorname{dom} \gamma \rightarrow \mathbb{Z}$ and $\varphi_{\text {ran }}: \operatorname{ran} \varphi \rightarrow \mathbb{Z}$. We put $\operatorname{dom} \kappa=\operatorname{dom} \gamma, \operatorname{ran} \kappa=\operatorname{dom} \varphi$ and $\kappa=\gamma_{\text {dom }} \cdot$ $\left(\varphi_{\text {dom }}\right)^{-1}$. Then $\kappa: \mathbb{Z} \rightarrow \mathbb{Z}$ is a monotone partial map as a composition of monotone partial maps. We define an injective partial map $\chi: \mathbb{Z} \rightarrow \mathbb{Z}$ in the following way: $\operatorname{dom} \chi=\mathbb{Z}, \operatorname{ran} \chi=\operatorname{ran} \gamma$ and $(n) \chi=(n)\left(\left(\varphi_{\text {ran }}\right)^{-1} \cdot \varphi^{-1} \cdot \kappa \cdot \gamma\right)$ for $n \in \mathbb{Z}$. Then $\chi: \mathbb{Z} \rightarrow \mathbb{Z}$ is a monotone partial map, being a composition of monotone partial maps. We put $\operatorname{dom} \xi=\operatorname{ran} \varphi, \operatorname{ran} \xi=\operatorname{ran} \gamma$ and $\xi=\varphi_{\operatorname{ran}} \cdot \chi$. Then $\xi: \mathbb{Z} \rightarrow \mathbb{Z}$ is a monotone partial map, being a composition of monotone partial maps. Hence $\gamma=\kappa \cdot \varphi \cdot \xi$ and so $\mathscr{I}_{\infty}^{\nearrow}(\mathbb{Z})$ is simple.

$(i x)$ Let $\varepsilon, \varphi \in E\left(\mathscr{I}_{\infty}^{\nearrow}(\mathbb{Z})\right)$ be arbitrary. Then by statement $(i)$ we have that dom $\varepsilon=\operatorname{ran} \varepsilon$ and $\operatorname{dom} \varphi=\operatorname{ran} \varphi$. Since the sets $\mathbb{Z} \backslash \operatorname{dom} \varepsilon$ and $\mathbb{Z} \backslash \operatorname{dom} \varphi$ are finite and the sets $\operatorname{dom} \varepsilon$ and $\operatorname{dom} \varphi$ are order-isomorphic to $\mathbb{Z}$ we conclude that there exist bijective monotone maps $\varepsilon_{\text {dom }}$ : dom $\varepsilon \rightarrow \mathbb{Z}$ and $\varphi_{\text {dom }}: \operatorname{dom} \varphi \rightarrow \mathbb{Z}$. Also, we note that for every integer $k$ the translation $\sigma_{k}: \mathbb{Z} \rightarrow \mathbb{Z}: n \mapsto n+k$ is a bijective monotone map. Now we define for any integer $i$

$$
\alpha_{i}=\varepsilon_{\mathrm{dom}} \cdot \sigma_{i} \cdot\left(\varphi_{\mathrm{dom}}\right)^{-1} \text {. }
$$

Then we have that

$$
\begin{aligned}
\alpha_{i} \cdot \alpha_{i}^{-1} & =\varepsilon_{\mathrm{dom}} \cdot \sigma_{i} \cdot\left(\varphi_{\mathrm{dom}}\right)^{-1} \cdot \varphi_{\mathrm{dom}} \cdot \sigma_{i}^{-1} \cdot\left(\varepsilon_{\mathrm{dom}}\right)^{-1}= \\
& =\varepsilon_{\mathrm{dom}} \cdot \sigma_{i} \cdot \mathbb{I} \cdot \sigma_{i}^{-1} \cdot\left(\varepsilon_{\mathrm{dom}}\right)^{-1}=\varepsilon_{\mathrm{dom}} \cdot \sigma_{i} \cdot \sigma_{i}^{-1} \cdot\left(\varepsilon_{\mathrm{dom}}\right)^{-1}= \\
& =\varepsilon_{\mathrm{dom}} \cdot \mathbb{I} \cdot\left(\varepsilon_{\mathrm{dom}}\right)^{-1}=\varepsilon_{\mathrm{dom}} \cdot\left(\varepsilon_{\mathrm{dom}}\right)^{-1}= \\
& =\varepsilon
\end{aligned}
$$

and

$$
\begin{aligned}
\alpha_{i}^{-1} \cdot \alpha_{i} & =\varphi_{\mathrm{dom}} \cdot \sigma_{i}^{-1} \cdot\left(\varepsilon_{\mathrm{dom}}\right)^{-1} \cdot \varepsilon_{\mathrm{dom}} \cdot \sigma_{i} \cdot\left(\varphi_{\mathrm{dom}}\right)^{-1}= \\
& =\varphi_{\mathrm{dom}} \cdot \sigma_{i}^{-1} \cdot \mathbb{I} \cdot \sigma_{i} \cdot\left(\varphi_{\mathrm{dom}}\right)^{-1}=\varphi_{\mathrm{dom}} \cdot \sigma_{i}^{-1} \cdot \sigma_{i} \cdot\left(\varphi_{\mathrm{dom}}\right)^{-1}= \\
& =\varphi_{\mathrm{dom}} \cdot \mathbb{I} \cdot\left(\varphi_{\mathrm{dom}}\right)^{-1}=\varphi_{\mathrm{dom}} \cdot\left(\varphi_{\mathrm{dom}}\right)^{-1}= \\
& =\varphi
\end{aligned}
$$

for every integer $i$. This completes the proof of the assertion.

Proposition 2.2. The group of units $H(\mathbb{I})$ of the semigroup $\mathscr{I}_{\infty}^{\nearrow}(\mathbb{Z})$ is isomorphic to $\mathbb{Z}(+)$.

Proof. Let $\alpha$ be an arbitrary element of $H(\mathbb{I})$. Then $\alpha$ is a bijective monotone map from $\mathbb{Z}$ onto $\mathbb{Z}$. We fix arbitrary $n \in \mathbb{Z}$. Then the monotonicity of $\alpha$ implies that $(n) \alpha<(n+1) \alpha$. If $(n) \alpha+1<(n+1) \alpha$ then there exists an integer $m$ such that $(m) \alpha=(n) \alpha+1$. But if $m>n+1$ or $m<n$ this contradicts the monotonicity of $\alpha$. Therefore we get that $(n) \alpha+1=(n+1) \alpha$. Similarly we get that $(n) \alpha-1=(n-1) \alpha$. Hence every $\alpha \in H(\mathbb{I})$ is a shift of the set of integers. We define the map $h: H(\mathbb{I}) \rightarrow \mathbb{Z}(+)$ by the formula $(\alpha) h=(n) \alpha-n$. Since $\alpha$ is a shift of the set of integers we conclude that the definition of the map $h$ is correct. Simple verifications show that $h: H(\mathbb{I}) \rightarrow \mathbb{Z}(+)$ is a group isomorphism.

Since $\mathscr{I}_{\infty}^{\nearrow}(\mathbb{Z})$ is an inverse semigroup, Proposition 2.1] $(i x)$ and Lemma 1.1 from [24] imply the following:

Proposition 2.3. $\mathscr{I}_{\infty}^{\nearrow}(\mathbb{Z})$ is a bisimple semigroup.

Theorem 2.20 of [9], and our Propositions 2.2 and 2.3 imply the following corollary: 
Corollary 2.4. Every maximal subgroup of the semigroup $\mathscr{I}_{\infty}^{\nearrow}(\mathbb{Z})$ is isomorphic to $\mathbb{Z}(+)$.

Proposition 2.5. For every $\alpha, \beta \in \mathscr{I}_{\infty}^{\nearrow}(\mathbb{Z})$, both sets $\left\{\chi \in \mathscr{I}_{\infty}^{\nearrow}(\mathbb{Z}) \mid \alpha \cdot \chi=\beta\right\}$ and $\left\{\chi \in \mathscr{I}_{\infty}^{\nearrow}(\mathbb{Z}) \mid\right.$ $\chi \cdot \alpha=\beta\}$ are finite.

Proof. We denote $A=\left\{\chi \in \mathscr{I}_{\infty}^{\nearrow}(\mathbb{Z}) \mid \alpha \cdot \chi=\beta\right\}$ and $B=\left\{\chi \in \mathscr{I}_{\infty}^{\nearrow}(\mathbb{Z}) \mid \alpha^{-1} \cdot \alpha \cdot \chi=\alpha^{-1} \cdot \beta\right\}$. Then $A \subseteq B$ and the restriction of any partial map $\chi \in B$ to $\operatorname{dom}\left(\alpha^{-1} \cdot \alpha\right)$ coincides with the partial map $\alpha^{-1} \cdot \beta$. Since every partial map from $\mathscr{I}_{\infty}^{\nearrow}(\mathbb{Z})$ is monotone we conclude that the set $B$ is finite and hence so is $A$.

Lemma 2.6. Let $S$ be an arbitrary semigroup and $h: \mathscr{I}_{\infty}^{\nearrow}(\mathbb{Z}) \rightarrow S$ a semigroup homomorphism. If there exist distinct idempotents $\varepsilon, \varphi \in \mathscr{I}_{\infty}^{\nearrow}(\mathbb{Z})$ such that $(\varepsilon) h=(\varphi) h$ then $(\psi) h=(\mathbb{I}) h$ for all $\psi \in E\left(\mathscr{I}_{\infty}^{\nearrow}(\mathbb{Z})\right)$.

Proof. Since $(\varepsilon) h=(\varphi) h=(\varphi \cdot \varphi) h=(\varphi) h \cdot(\varphi) h=(\varphi) h \cdot(\varepsilon) h=(\varphi \cdot \varepsilon) h$ we can assume without loss of generality that $\varepsilon \leqslant \varphi$ in $E\left(\mathscr{I}_{\infty}^{\nearrow}(\mathbb{Z})\right)$. Therefore, if $\iota$ is an idempotent of the semigroup $\mathscr{I}_{\infty}^{\nearrow}(\mathbb{Z})$ such that $\varepsilon \leqslant \iota \leqslant \varphi$ then $(\varepsilon) h=(\iota) h$. Hence Proposition 2.1 (ii) implies that we can assume without loss of generality that $|\operatorname{dom} \varphi \backslash \operatorname{dom} \varepsilon|=1$.

Let $\psi$ let be an arbitrary idempotent of the semigroup $\mathscr{I}_{\infty}^{\nearrow}(\mathbb{Z})$ and $n_{0}=\min \{\mathbb{Z} \backslash \operatorname{dom} \psi\}-1$. Let $\sigma: \mathbb{Z} \rightarrow \mathbb{Z}$ be a partial order preserving injective map which maps $\operatorname{dom} \varphi$ onto $\mathbb{Z}$ and $n=\mathbb{Z} \backslash(\operatorname{dom} \varepsilon) \sigma$. Without loss of generality we can assume that $n=n_{0}$. Then $\widetilde{\varphi}=\sigma^{-1} \circ \varphi \circ \sigma: \mathbb{Z} \rightarrow \mathbb{Z}$ is an identity map and $\widetilde{\varepsilon}=\sigma^{-1} \circ \varepsilon \circ \sigma$ is an identity map from $\mathbb{Z} \backslash\left\{n_{0}\right\}$ onto $\mathbb{Z} \backslash\left\{n_{0}\right\}$. Then $\widetilde{\varepsilon}$ is a unit of the semigroup $\mathscr{I}_{\infty}^{\nearrow}(\mathbb{Z})$. Since $\sigma \in \mathscr{I}_{\infty}^{\nearrow}(\mathbb{Z})$ and $\operatorname{dom} \varepsilon \varsubsetneqq \operatorname{dom} \varphi$ we have that

$$
\begin{aligned}
& (\widetilde{\varphi}) h=(\sigma \cdot \varphi \cdot \sigma) h=(\sigma) h \cdot(\varphi) h \cdot(\sigma) h=(\sigma) h \cdot(\varepsilon) h \cdot(\sigma) h=(\widetilde{\varepsilon}) h, \\
\widetilde{\varepsilon} \cdot \widetilde{\varphi}= & \left(\sigma^{-1} \cdot \varepsilon \cdot \sigma\right) \cdot\left(\sigma^{-1} \cdot \varphi \cdot \sigma\right)=\sigma^{-1} \cdot \varepsilon \cdot\left(\sigma \cdot \sigma^{-1}\right) \cdot \varphi \cdot \sigma=\sigma^{-1} \cdot \varepsilon \cdot \varphi \cdot \varphi \cdot \sigma= \\
= & \sigma^{-1} \cdot \varepsilon \cdot \varphi \cdot \sigma=\sigma^{-1} \cdot \varepsilon \cdot \sigma=\widetilde{\varepsilon}, \\
\widetilde{\varphi} \cdot \widetilde{\varepsilon}= & \left(\sigma^{-1} \cdot \varphi \cdot \sigma\right) \cdot\left(\sigma^{-1} \cdot \varepsilon \cdot \sigma\right)=\sigma^{-1} \cdot \varphi \cdot\left(\sigma \cdot \sigma^{-1}\right) \cdot \varepsilon \cdot \sigma=\sigma^{-1} \cdot \varphi \cdot \varphi \cdot \varepsilon \cdot \sigma= \\
= & \sigma^{-1} \cdot \varphi \cdot \varepsilon \cdot \sigma=\sigma^{-1} \cdot \varepsilon \cdot \sigma=\widetilde{\varepsilon},
\end{aligned}
$$

and hence $\widetilde{\varepsilon} \leqslant \widetilde{\varphi}$.

We observe that $\widetilde{\varphi}, \widetilde{\varepsilon} \in \mathscr{C}\left(n_{0},+\right)$. Since $(\widetilde{\varphi}) h=(\widetilde{\varepsilon}) h$, Corollary $1.32[9]$ implies that $(\widetilde{\varphi}) h=(\chi) h$ for every idempotent $\chi \in \mathscr{C}\left(n_{0},+\right)$. Since $i>n_{0}$ for all $i \in \mathbb{Z} \backslash$ dom $\psi$, the definition of the semigroup $\mathscr{I}_{\infty}^{\nearrow}(\mathbb{Z})$ implies that there exists an idempotent $\varepsilon_{0} \in \mathscr{C}\left(n_{0},+\right)$ such that $\varepsilon_{0} \leqslant \psi \leqslant \widetilde{\varphi}$. Therefore we have that $(\psi) h=\left(\varepsilon_{0}\right) h=(\widetilde{\varphi}) h$. This completes the proof of the lemma.

Theorem 2.7. Let $S$ be a semigroup and $h: \mathscr{I}_{\infty}^{\nearrow}(\mathbb{Z}) \rightarrow S$ a non-annihilating homomorphism. Then either $h$ is a monomorphism or $\left(\mathscr{I}_{\infty}^{\nearrow}(\mathbb{Z})\right) h$ is a subgroup of $S$.

Proof. Suppose that $h: \mathscr{I}_{\infty}^{\nearrow}(\mathbb{Z}) \rightarrow S$ is not a monomorphism. Then $(\alpha) h=(\beta) h$, for some distinct $\alpha, \beta \in \mathscr{I}_{\infty}^{\nearrow}(\mathbb{Z})$. We consider two cases:

(i) $\alpha$ and $\beta$ are not $\mathscr{H}$-equivalent;

(ii) $\alpha$ and $\beta$ are $\mathscr{H}$-equivalent.

Suppose that case $(i)$ holds. Since $\mathscr{I}_{\infty}^{\nearrow}(\mathbb{Z})$ is an inverse semigroup we have that either $\alpha \cdot \alpha^{-1} \neq \beta \cdot \beta^{-1}$ or $\alpha^{-1} \cdot \alpha \neq \beta^{-1} \cdot \beta$. Suppose that $\alpha \cdot \alpha^{-1} \neq \beta \cdot \beta^{-1}$. In the other case the proof is similar. Since $\mathscr{I}_{\infty}^{\nearrow}(\mathbb{Z})$ is an inverse semigroup we conclude that

$$
\left(\alpha^{-1}\right) h=((\alpha) h)^{-1}=((\beta) h)^{-1}=\left(\beta^{-1}\right) h
$$

and hence $\left(\alpha \cdot \alpha^{-1}\right) h=(\alpha) h \cdot\left(\alpha^{-1}\right) h=(\beta) h \cdot\left(\beta^{-1}\right) h=\left(\beta \cdot \beta^{-1}\right) h$. Therefore the assertion of Lemma 2.6 holds. Since every homomorphic image of an inverse semigroup is an inverse semigroup we conclude that $\left(\mathscr{I}_{\infty}^{\nearrow}(\mathbb{Z})\right) h$ is a subgroup of $S$.

Suppose that $\alpha \mathscr{H} \beta$. Then by Theorem 2.20 of [9] there exist distinct $\alpha_{0}, \beta_{0} \in H(\mathbb{I})$ such that $\left(\alpha_{0}\right) h=\left(\beta_{0}\right) h$. Therefore we have that $(\mathbb{I}) h=(\gamma) h$ for $\gamma=\alpha_{0}^{-1} \cdot \beta_{0} \in H(\mathbb{I})$ and $\gamma \neq \mathbb{I}$. We fix an arbitrary integer $i$. Let $\iota: \mathbb{Z} \backslash\{i\} \rightarrow \mathbb{Z} \backslash\{i\}$ be an identity map. Then $(\iota) h=(\iota \cdot \mathbb{I}) h=(\iota \cdot \gamma) h$. Hence $\iota$ is an idempotent of the semigroup $\mathscr{I}_{\infty}^{\nearrow}(\mathbb{Z})$ and $\operatorname{ran} \iota \neq \operatorname{ran}(\iota \cdot \gamma)$. Therefore by Proposition 2.1 (vii) 
the elements $\iota$ and $\iota \cdot \gamma$ are not $\mathscr{H}$-equivalent in the semigroup $\mathscr{I} \mathscr{\infty}^{\nearrow}(\mathbb{Z})$. This implies that there exist distinct non- $\mathscr{H}$-equivalent elements $\alpha, \beta$ in $\mathscr{I}_{\infty}^{\rightarrow}(\mathbb{Z})$ such that $(\alpha) h=(\beta) h$ and hence case $(i)$ holds. Therefore we get that $\left(\mathscr{I}_{\infty}^{\rightarrow}(\mathbb{Z})\right) h$ is a subgroup of $S$.

Proposition 2.8. Let $\mathfrak{C}_{m g}$ be a least group congruence on the semigroup $\mathscr{I}_{\infty}^{\nearrow}(\mathbb{Z})$. Then the quotient semigroup $\mathscr{I}_{\infty}^{\nearrow}(\mathbb{Z}) / \mathfrak{C}_{m g}$ is isomorphic to the direct product $\mathbb{Z}(+) \times \mathbb{Z}(+)$.

Proof. Let $\alpha$ and $\beta$ be $\mathfrak{C}_{m g}$-equivalent elements of the semigroup $\mathscr{I}_{\infty}^{\nearrow}(\mathbb{Z})$. Then by Lemma III.5.2 from [26] there exists an idempotent $\varepsilon_{0}$ in $\mathscr{I}_{\infty}^{\nearrow}(\mathbb{Z})$ such that $\alpha \cdot \varepsilon_{0}=\beta \cdot \varepsilon_{0}$. Since $\mathscr{I}_{\infty}^{\nearrow}(\mathbb{Z})$ is an inverse semigroup we conclude that $\alpha \cdot \varepsilon=\beta \cdot \varepsilon$ for all $\varepsilon \in E\left(\mathscr{I}_{\infty}^{\nearrow}(\mathbb{Z})\right)$ such that $\varepsilon \leqslant \varepsilon_{0}$. Then Lemma 1.1 implies that there exist integers $d$ and $u$ such that

$$
\begin{array}{llrl}
(m-1) \alpha & =(m) \alpha-1, & & (n+1) \alpha=(n) \alpha+1, \\
(m-1) \beta & =(m) \beta-1, & & (n+1) \beta=(n) \beta+1,
\end{array}
$$

for all integers $m \leqslant d$ and $n \geqslant u$. We put $D=\min \{(d) \alpha,(d) \beta\}$ and $U=\max \{(u) \alpha,(u) \beta\}$. Let $\varepsilon_{1}$ be an identity map from $\mathbb{Z} \backslash\{D, D+1, \ldots, U\}$ onto itself. Then $\varepsilon^{0}=\varepsilon_{1} \circ \varepsilon_{0} \leqslant \varepsilon_{0}$ and hence we have that $\alpha \cdot \varepsilon^{0}=\beta \cdot \varepsilon^{0}$. Therefore we have showed that if the elements $\alpha$ and $\beta$ of the semigroup $\mathscr{I}_{\infty}^{\nearrow}(\mathbb{Z})$ are $\mathfrak{C}_{m g}$-equivalent, then there exist integers $d$ and $u$ such that

$$
(m) \alpha=(m) \beta \quad \text { and } \quad(n) \alpha=(n) \beta,
$$

for all integers $m \leqslant d$ and $n \geqslant u$.

Conversely, suppose that exist integers $d$ and $u$ such that

$$
(m) \alpha=(m) \beta \quad \text { and } \quad(n) \alpha=(n) \beta,
$$

for all integers $m \leqslant d$ and $n \geqslant u$. Then we have that $d \leqslant u$. If $d=u$ or $d=u-1$ then $\alpha=\beta$ in $\mathscr{I}_{\infty}^{\nearrow}(\mathbb{Z})$ and hence $\alpha$ and $\beta$ are $\mathfrak{C}_{m g}$-equivalent. If $d<u-1$ then we put $\varepsilon_{0}$ to be the identity map of the set $\mathbb{Z} \backslash\{(d+1) \alpha, \ldots,(u-1) \alpha\}$. Then we get that $(n)\left(\alpha \circ \varepsilon_{0}\right)=(n)\left(\beta \circ \varepsilon_{0}\right)$ for any $n \in \mathbb{Z} \backslash\{d+1, \ldots, u-1\}$ and therefore $\alpha \cdot \varepsilon_{0}=\beta \cdot \varepsilon_{0}$. Hence Lemma III.5.2 from [26] implies that $\alpha$ and $\beta$ are $\mathfrak{C}_{m g}$-equivalent elements of the semigroup $\mathscr{I}_{\infty}^{\nearrow}(\mathbb{Z})$.

Now we define the map $h: \mathscr{I}_{\infty}^{\nearrow}(\mathbb{Z}) \rightarrow \mathbb{Z}(+) \times \mathbb{Z}(+)$ by the formula

$$
(\alpha) h=\left(\left(d_{\alpha}\right) \alpha-d_{\alpha},\left(u_{\alpha}\right) \alpha-u_{\alpha}\right),
$$

where the integers $d_{\alpha}$ and $u_{\alpha}$ are defined in Lemma 1.1.

We observe that

$$
\left(d_{\alpha}-n\right) \alpha=\left(d_{\alpha}\right) \alpha-n \quad \text { and } \quad\left(u_{\alpha}+n\right) \alpha=\left(u_{\alpha}\right) \alpha+n,
$$

for any positive integer $n$. Hence we have that

$$
(m) \alpha-m=\left(d_{\alpha}\right) \alpha-d_{\alpha} \quad \text { and } \quad(n) \alpha-n=\left(u_{\alpha}\right) \alpha-u_{\alpha},
$$

for all integers $m \leqslant d_{\alpha}$ and $n \geqslant u_{\alpha}$.

Lemma 1.1 implies that there exist integers $d^{0}$ and $u^{0}$ such that

$$
\begin{aligned}
(m-1) \alpha & =(m) \alpha-1, & (n+1) \alpha & =(n) \alpha+1, \\
(m-1) \beta & =(m) \beta-1, & (n+1) \beta & =(n) \beta+1, \\
(m-1)(\alpha \cdot \beta) & =(m)(\alpha \cdot \beta)-1, & (n+1)(\alpha \cdot \beta) & =(n)(\alpha \cdot \beta)+1,
\end{aligned}
$$

for all integers $m \leqslant d^{0}$ and $n \geqslant u^{0}$. Hence for all integers $m \leqslant d^{0}$ and $n \geqslant u^{0}$ we have that

$$
\begin{gathered}
(m)(\alpha \cdot \beta)-m=(m)(\alpha \cdot \beta)-(m) \alpha+(m) \alpha-m=\left(\left(d_{\beta}\right) \beta-d_{\beta}\right)+\left(\left(d_{\alpha}\right) \alpha-d_{\alpha}\right), \\
(n)(\alpha \cdot \beta)-n=(n)(\alpha \cdot \beta)-(n) \alpha+(n) \alpha-n=\left(\left(u_{\beta}\right) \beta-u_{\beta}\right)+\left(\left(u_{\alpha}\right) \alpha-u_{\alpha}\right) .
\end{gathered}
$$

This implies that the map $h: \mathscr{I}_{\infty}^{\nearrow}(\mathbb{Z}) \rightarrow \mathbb{Z}(+) \times \mathbb{Z}(+)$ is a homomorphism.

Theorem 2.7 and Proposition 2.8 imply the following:

Theorem 2.9. Let $S$ be a semigroup and $h: \mathscr{I}_{\infty}^{\nearrow}(\mathbb{Z}) \rightarrow S$ a non-annihilating homomorphism. Then either $h$ is a monomorphism or $\left(\mathscr{I}_{\infty}^{\nearrow}(\mathbb{Z})\right) h$ is a homomorphic image of the group $\mathbb{Z}(+) \times \mathbb{Z}(+)$. 


\section{Some REMARKS ON THE SEMigRoup $\mathscr{I}_{\infty}^{\rightarrow}(\mathbb{Z})$}

In this section we shall denote the identity of the semigroup $\mathscr{I}_{\infty}^{\rightarrow}(\mathbb{Z})$ by $\mathbb{I}$ and the group of units of $\mathscr{I}_{\infty}^{\rightarrow}(\mathbb{Z})$ by $H(\mathbb{I})$. The proof of the following proposition is similar to corresponding propositions in Section 2 .

Proposition 3.1. $\quad(i) E\left(\mathscr{I}_{\infty}^{\text {q }}(\mathbb{Z})\right)=E\left(\mathscr{I}_{\infty}^{\nearrow}(\mathbb{Z})\right)$.

(ii) $\alpha \mathscr{R} \beta$ in $\mathscr{I}_{\infty}^{\rightarrow}(\mathbb{Z})$ if and only if $\operatorname{dom} \alpha=\operatorname{dom} \beta$.

(iii) $\alpha \mathscr{L} \beta$ in $\mathscr{I}_{\infty}^{\rightarrow}(\mathbb{Z})$ if and only if $\operatorname{ran} \alpha=\operatorname{ran} \beta$.

(iv) $\alpha \mathscr{H} \beta$ in $\mathscr{I}_{\infty}^{\rightarrow}(\mathbb{Z})$ if and only if $\operatorname{dom} \alpha=\operatorname{dom} \beta$ and $\operatorname{ran} \alpha=\operatorname{ran} \beta$.

$(v) \mathscr{I}_{\infty}^{\rightarrow}(\mathbb{Z})$ is a simple semigroup and hence $\mathscr{J}=\mathscr{I}_{\infty}^{\rightarrow+}(\mathbb{Z}) \times \mathscr{I}_{\infty}^{\rightarrow+}(\mathbb{Z})$.

(vi) For all idempotents $\varepsilon, \varphi \in \mathscr{I}_{\infty}^{\rightarrow}(\mathbb{Z})$ there exist infinitely many elements $\alpha, \beta \in \mathscr{I}_{\infty}^{\rightarrow}(\mathbb{Z})$ such that $\alpha \cdot \beta=\varepsilon$ and $\beta \cdot \alpha=\varphi$.

(vii) $\mathscr{I}_{\infty}^{\rightarrow}(\mathbb{Z})$ is a bisimple semigroup.

(viii) For all $\alpha, \beta \in \mathscr{I}_{\infty}^{\rightarrow}(\mathbb{Z})$, both sets $\left\{\chi \in \mathscr{I}_{\infty}^{\rightarrow}(\mathbb{Z}) \mid \alpha \cdot \chi=\beta\right\}$ and $\left\{\chi \in \mathscr{I}_{\infty}^{\rightarrow}(\mathbb{Z}) \mid \chi \cdot \alpha=\beta\right\}$ are finite.

Proposition 3.2. For every $\alpha \in \mathscr{I}_{\infty}^{\rightarrow}(\mathbb{Z})$ there exist idempotents $\varepsilon_{l}, \varepsilon_{r}, \varepsilon$ in $\mathscr{I}_{\infty}^{\rightarrow}(\mathbb{Z})$ such that $\varepsilon_{l} \cdot \alpha, \alpha \cdot$ $\varepsilon_{r}, \varepsilon \cdot \alpha \cdot \varepsilon \in \mathscr{I}_{\infty}^{\nearrow}(\mathbb{Z})$.

Proof. The definition of the semigroup $\mathscr{I}_{\infty}^{\rightarrow+}(\mathbb{Z})$ implies that for every element $\alpha$ of $\mathscr{I}_{\infty}^{q}(\mathbb{Z})$ there exists a smallest finite (or empty) subset $F_{\alpha}$ such that the restriction $\left.\alpha\right|_{\operatorname{dom} \alpha \backslash F_{\alpha}}: \mathbb{Z} \rightarrow \mathbb{Z}$ is a monotone partial map. We put $\varepsilon_{l}=\operatorname{id}_{\operatorname{dom} \alpha \backslash F_{\alpha}}$ to be the identity map from $\operatorname{dom} \alpha \backslash F_{\alpha}$ onto $\operatorname{dom} \alpha \backslash F_{\alpha}$. Also we set $\varepsilon_{r}=\operatorname{id}_{\left(\operatorname{dom} \alpha \backslash F_{\alpha}\right) \alpha}$ and $\varepsilon=\varepsilon_{l} \cdot \varepsilon_{r}$. Then we have that $\varepsilon_{l} \cdot \alpha, \alpha \cdot \varepsilon_{r}, \varepsilon \cdot \alpha \cdot \varepsilon \in \mathscr{I}_{\infty}^{\nearrow}(\mathbb{Z})$.

We denote by $S_{\infty}(\mathbb{Z})$ the group of all bijective transformations of $\mathbb{Z}$ with finite supports (i.e., $\alpha \in$ $S_{\infty}(\mathbb{Z})$ if and only if the set $\{x \in \mathbb{Z} \mid(x) \alpha \neq x\}$ is finite). We observe that $S_{\infty}(\mathbb{Z})$ is a subgroup of the group of units $H(\mathbb{I})$ of the semigroup $\mathscr{I}_{\infty}^{\rightarrow}(\mathbb{Z})$ and since every element $\alpha$ in $H(\mathbb{I})$ is an almost monotone bijective selfmap of the set of integers we get that for every $\alpha \in H(\mathbb{I})$ there exists an integer $n_{\alpha}$ such that the set $\left\{i \in \mathbb{Z} \mid(i) \alpha+n_{\alpha} \neq i\right\}$ is finite. This observation implies that $S_{\infty}(\mathbb{Z})$ is a normal subgroup of $H(\mathbb{I})$. Moreover, we have that every element of the group of units $H(\mathbb{I})$ has a unique representation $\alpha=\sigma \cdot \beta$ by the formula $(n) \alpha=(n) \sigma+\beta, n \in \mathbb{Z}$, where $\sigma \in \alpha \in S_{\infty}(\mathbb{Z})$ and $\beta \in \mathbb{Z}(+)$. Hence we have that $H(\mathbb{I})=S_{\infty}(\mathbb{Z}) \cdot \mathbb{Z}(+)$ and it is obvious that $S_{\infty}(\mathbb{Z}) \cap \mathbb{Z}(+)=\{\mathbb{I}\}$. Thus the group $\mathbb{Z}(+)$ acts on $S_{\infty}(\mathbb{Z})$ by the conjugation action in $H(\mathbb{I})$ and hence it follows by Exercise 2.5.3 from [12] that the group $H(\mathbb{I})$ is isomorphic to the semidirect product $S_{\infty}(\mathbb{Z}) \rtimes \mathbb{Z}(+)$ (or split extension of $S_{\infty}(\mathbb{Z})$ by $\mathbb{Z}(+))$. Also, we observe that since the action of the group $\mathbb{Z}(+)$ on $S_{\infty}(\mathbb{Z})$ is not the identity map we conclude that the group $H(\mathbb{I})$ is not isomorphic to the direct product $S_{\infty}(\mathbb{Z}) \times \mathbb{Z}(+)$. We put $\widetilde{\mathbb{Z}}(+)=S_{\infty}(\mathbb{Z}) \rtimes \mathbb{Z}(+)$. Therefore we have proved the following:

Proposition 3.3. The group of units $H(\mathbb{I})$ of the semigroup $\mathscr{I}_{\infty}^{\rightarrow+}(\mathbb{Z})$ is isomorphic to $\widetilde{\mathbb{Z}}(+)$.

Proposition 3.1 (vii) and Theorem 2.20 of [9] imply the following corollary:

Corollary 3.4. Every maximal subgroup of the semigroup $\mathscr{I}_{\infty}^{\rightarrow}(\mathbb{Z})$ is isomorphic to $\widetilde{\mathbb{Z}}(+)$.

Theorem 3.5. Let $S$ be a semigroup and $h: \mathscr{I}_{\infty}^{\rightarrow \rightarrow}(\mathbb{Z}) \rightarrow S$ a non-annihilating homomorphism. Then either $h$ is a monomorphism or $\left(\mathscr{I}_{\infty}^{\rightarrow}(\mathbb{Z})\right) h$ is a subgroup of $S$.

Proof. Suppose that $h: \mathscr{I}_{\infty}^{\rightarrow}(\mathbb{Z}) \rightarrow S$ is not an monomorphism. Then $(\alpha) h=(\beta) h$, for some distinct $\alpha, \beta \in \mathscr{I}_{\infty}^{\rightarrow}(\mathbb{Z})$.

Suppose that $\alpha$ and $\beta$ are not $\mathscr{H}$-equivalent. Since $\mathscr{I}_{\infty}^{+}(\mathbb{Z})$ is an inverse semigroup, we have that either $\alpha \cdot \alpha^{-1} \neq \beta \cdot \beta^{-1}$ or $\alpha^{-1} \cdot \alpha \neq \beta^{-1} \cdot \beta$. Suppose that $\alpha \cdot \alpha^{-1} \neq \beta \cdot \beta^{-1}$. Since $\mathscr{I}_{\infty}^{\rightarrow}(\mathbb{Z})$ is an inverse semigroup, we conclude that $\left(\alpha^{-1}\right) h=\left(\beta^{-1}\right) h$ and hence $\left(\alpha \cdot \alpha^{-1}\right) h=\left(\beta \cdot \beta^{-1}\right) h$. Therefore the assertion of Lemma 2.6 holds for the subsemigroup $\mathscr{I}_{\infty}^{\nearrow}(\mathbb{Z})$ of the semigroup $\mathscr{I}_{\infty}^{\rightarrow}(\mathbb{Z})$. Now by Proposition $3.1(i)$ and since every homomorphic image of an inverse semigroup is an inverse semigroup it follows that $\left(\mathscr{I}_{\infty}^{\rightarrow}(\mathbb{Z})\right) h$ is a subgroup of $S$. 
Suppose that $\alpha \mathscr{H} \beta$. Then by Theorem 2.20 of [9] there exist distinct $\alpha_{0}, \beta_{0} \in H(\mathbb{I})$ such that $\left(\alpha_{0}\right) h=\left(\beta_{0}\right) h$. Then we have that $(\mathbb{I}) h=(\gamma) h$ for $\gamma=\alpha_{0}^{-1} \cdot \beta_{0} \in H(\mathbb{I})$ and $\gamma \neq \mathbb{I}$. We fix an arbitrary integer $i$. Let $\iota: \mathbb{Z} \backslash\{i\} \rightarrow \mathbb{Z} \backslash\{i\}$ be the identity map. Hence $(\iota) h=(\iota \cdot \mathbb{I}) h=(\iota \cdot \gamma) h$. Then $\iota$ is an idempotent of the semigroup $\mathscr{I}_{\infty}^{\rightarrow}(\mathbb{Z})$ and $\operatorname{ran} \iota \neq \operatorname{ran}(\iota \cdot \gamma)$. Therefore by Proposition 3.1 (iv) the elements $\iota$ and $\iota \cdot \gamma$ are not $\mathscr{H}$-equivalent in the semigroup $\mathscr{I}_{\infty}^{\rightarrow}(\mathbb{Z})$. This implies that there exist distinct non- $\mathscr{H}$-equivalent elements $\alpha, \beta$ in $\mathscr{I}_{\infty}^{\rightarrow}(\mathbb{Z})$ such that $(\alpha) h=(\beta) h$ and hence $\left(\mathscr{I}_{\infty}^{\rightarrow}(\mathbb{Z})\right) h$ is a subgroup of $S$.

Proposition 3.6. Let $\mathfrak{C}_{m g}$ be a least group congruence on the semigroup $\mathscr{I}_{\infty}(\mathbb{Z})$. Then the quotient semigroup $\mathscr{I}_{\infty}^{\rightarrow}(\mathbb{Z}) / \mathfrak{C}_{m g}$ is isomorphic to the direct product $\mathbb{Z}(+) \times \mathbb{Z}(+)$.

Proof. Let $\alpha$ and $\beta$ be $\mathfrak{C}_{m g}$-equivalent elements of the semigroup $\mathscr{I}_{\infty} \rightarrow(\mathbb{Z})$. Then by Lemma III.5.2 from [26] there exists an idempotent $\varepsilon_{0}$ in $\mathscr{I}_{\infty}^{\rightarrow}(\mathbb{Z})$ such that $\alpha \cdot \varepsilon_{0}=\beta \cdot \varepsilon_{0}$. By Proposition 3.2 we can assume without loss of generality that $\alpha \cdot \varepsilon_{0}, \beta \cdot \varepsilon_{0} \in \mathscr{I}_{\infty}^{\nearrow}(\mathbb{Z})$. Then similarly as in the proof of Proposition 2.8 we can show that elements $\alpha$ and $\beta$ of the semigroup $\mathscr{I}_{\infty}^{\rightarrow}(\mathbb{Z})$ are $\mathfrak{C}_{m g}$-equivalent if and only if there exist integers $d$ and $u$ such that

$$
(m) \alpha=(m) \beta \quad \text { and } \quad(n) \alpha=(n) \beta,
$$

for all integers $m \leqslant d$ and $n \geqslant u$.

Let $\alpha_{0}=\alpha \cdot \varepsilon_{0}$. Then the map $h: \mathscr{I}_{\infty}^{\rightarrow}(\mathbb{Z}) \rightarrow \mathbb{Z}(+) \times \mathbb{Z}(+)$ defined by the formula

$$
(\alpha) h=\left(\left(d_{\alpha_{0}}\right) \alpha-d_{\alpha_{0}},\left(u_{\alpha_{0}}\right) \alpha-u_{\alpha_{0}}\right) \text {, }
$$

where the integers $d_{\alpha_{0}}$ and $u_{\alpha_{0}}$ are defined for element $\alpha_{0}$ of the semigroup $\mathscr{I}_{\infty}^{\nearrow}(\mathbb{Z})$ in Lemma 1.1 , is a natural homomorphism which is generated by the least group congruence $\mathfrak{C}_{m g}$ on the semigroup $\mathscr{I}_{\infty}^{\rightarrow}(\mathbb{Z})$.

Theorem 3.5 and Proposition 3.6 imply the following:

Theorem 3.7. Let $S$ be a semigroup and $h: \mathscr{I}_{\infty}^{\rightarrow}(\mathbb{Z}) \rightarrow S$ a non-annihilating homomorphism. Then either $h$ is a monomorphism or $\left(\mathscr{I}_{\infty}^{\rightarrow}(\mathbb{Z})\right) h$ is a homomorphic image of the group $\mathbb{Z}(+) \times \mathbb{Z}(+)$.

\section{On topologizations of The SEMigroup $\mathscr{I}_{\infty}^{\nearrow}(\mathbb{Z})$}

Theorem 4.1. Every Baire topology $\tau$ on $\mathscr{I}_{\infty}^{\nearrow}(\mathbb{Z})$ such that $\left(\mathscr{I}_{\infty}^{\nearrow}(\mathbb{Z}), \tau\right)$ is a Hausdorff semitopological semigroup is discrete.

Proof. If no point in $\mathscr{I}_{\infty}^{\nearrow}(\mathbb{Z})$ is isolated, then since $\left(\mathscr{I}_{\infty}^{\nearrow}(\mathbb{Z}), \tau\right)$ is Hausdorff, it follows that $\{\alpha\}$ is nowhere dense for all $\alpha \in \mathscr{I}_{\infty}^{\nearrow}(\mathbb{Z})$. But, if this is the case, then since $\mathscr{I}_{\infty}^{\nearrow}(\mathbb{Z})$ is countable it cannot be a Baire space. Hence $\mathscr{I}_{\infty}^{\nearrow}(\mathbb{Z})$ contains an isolated point $\mu$. If $\gamma \in \mathscr{I}_{\infty}^{\nearrow}(\mathbb{Z})$ is arbitrary, then by Proposition 2.1 (viii), there exist $\alpha, \beta \in \mathscr{I}_{\infty}^{\nearrow}(\mathbb{Z})$ such that $\alpha \cdot \gamma \cdot \beta=\mu$. The map $f: \chi \mapsto \alpha \cdot \chi \cdot \beta$ is continuous and so $(\{\mu\}) f^{-1}$ is open. By Proposition $\left[2.5,(\{\mu\}) f^{-1}\right.$ is finite and since $\left(\mathscr{I}_{\infty}^{\nearrow}(\mathbb{Z}), \tau\right)$ is Hausdorff, $\{\gamma\}$ is open, and hence isolated.

Since every Cech complete space (and hence every locally compact space) is Baire, Theorem 4.1 implies Corollaries 4.2 and 4.3 .

Corollary 4.2. Every Hausdorff Čech complete (locally compact) topology $\tau$ on $\mathscr{I}_{\infty}^{\nearrow}(\mathbb{Z})$ such that $\left(\mathscr{I}_{\infty}^{\nearrow}(\mathbb{Z}), \tau\right)$ is a Hausdorff semitopological semigroup is discrete.

Corollary 4.3. Every Hausdorff Baire topology (and hence Čech complete or locally compact topology) $\tau$ on $\mathscr{I}_{\infty}^{\nearrow}(\mathbb{Z})$ such that $\left(\mathscr{I}_{\infty}^{\nearrow}(\mathbb{Z}), \tau\right)$ is a Hausdorff topological semigroup is discrete.

The following example shows that there exists a non-discrete Tychonoff topology $\tau_{W}$ on the semigroup $\mathscr{I}_{\infty}^{\nearrow}(\mathbb{Z})$ such that $\left(\mathscr{I}_{\infty}^{\nearrow}(\mathbb{Z}), \tau_{W}\right)$ is a topological inverse semigroup.

Example 4.4. We define a topology $\tau_{W}$ on the semigroup $\mathscr{I}_{\infty}^{\nearrow}(\mathbb{Z})$ as follows. For every $\alpha \in \mathscr{I}_{\infty}^{\nearrow}(\mathbb{Z})$ we define a family

$$
\mathscr{B}_{W}(\alpha)=\left\{U_{\alpha}(F) \mid F \text { is a finite subset of } \operatorname{dom} \alpha\right\},
$$


where

$$
U_{\alpha}(F)=\left\{\beta \in \mathscr{I}_{\infty}^{\nearrow}(\mathbb{Z}) \mid \operatorname{dom} \beta \subseteq \operatorname{dom} \alpha \text { and }(x) \beta=(x) \alpha \text { for all } x \in F\right\} .
$$

It is straightforward to verify that $\left\{\mathscr{B}_{W}(\alpha)\right\}_{\alpha \in \mathscr{I}_{\infty}^{\nearrow}(\mathbb{Z})}$ forms a basis for a topology $\tau_{W}$ on the semigroup $\mathscr{I}_{\infty}^{\nearrow}(\mathbb{Z})$

Proposition 4.5. $\left(\mathscr{I}_{\infty}^{\nearrow}(\mathbb{Z}), \tau_{W}\right)$ is a Tychonoff topological inverse semigroup.

Proof. Let $\alpha$ and $\beta$ be arbitrary elements of the semigroup $\mathscr{I}_{\infty}^{\nearrow}(\mathbb{Z})$. We put $\gamma=\alpha \cdot \beta$ and let $F=\left\{n_{1}, \ldots, n_{i}\right\}$ be a finite subset of $\operatorname{dom} \gamma$. We denote $m_{1}=\left(n_{1}\right) \alpha, \ldots, m_{i}=\left(n_{i}\right) \alpha$ and $k_{1}=$ $\left(n_{1}\right) \gamma, \ldots, k_{i}=\left(n_{i}\right) \gamma$. Then we get that $\left(m_{1}\right) \beta=k_{1}, \ldots,\left(m_{i}\right) \beta=k_{i}$. Hence we have that

$$
U_{\alpha}\left(\left\{n_{1}, \ldots, n_{i}\right\}\right) \cdot U_{\beta}\left(\left\{m_{1}, \ldots, m_{i}\right\}\right) \subseteq U_{\gamma}\left(\left\{n_{1}, \ldots, n_{i}\right\}\right)
$$

and

$$
\left(U_{\gamma}\left(\left\{n_{1}, \ldots, n_{i}\right\}\right)\right)^{-1} \subseteq U_{\gamma^{-1}}\left(\left\{k_{1}, \ldots, k_{i}\right\}\right) .
$$

Therefore the semigroup operation and the inversion are continuous in $\left(\mathscr{I}_{\infty}^{\nearrow}(\mathbb{Z}), \tau_{W}\right)$.

Let $Z=\mathbb{Z} \cup\{a\}$ for some $a \notin \mathbb{Z}$. Then $Z^{Z}$ with the operation composition is a semigroup and the $\operatorname{map} \Psi: \mathscr{I}_{\infty}^{\nearrow}(\mathbb{Z}) \rightarrow Z^{Z}$ defined by the formula

$$
(x)(\alpha) \Psi= \begin{cases}(x) \alpha, & \text { if } x \in \operatorname{dom} \alpha \\ a, & \text { if } x \notin \operatorname{dom} \alpha\end{cases}
$$

is a monomorphism. Hence $Z^{Z}$ is a topological semigroup with the product topology if $Z$ has the discrete topology. Obviously, this topology generates topology $\tau_{W}$ on $\mathscr{I}_{\infty}^{\nearrow}(\mathbb{Z})$. Therefore by Theorem 2.3.11 from [14] topological space $Z^{Z}$ is Tychonoff and hence by Theorem 2.1.6 from [14] so is $\left(\mathscr{I}_{\infty}^{\nearrow}(\mathbb{Z}), \tau_{W}\right)$. This completes the proof of the proposition.

Theorem 4.6. Let $S$ be a topological semigroup which contains $\mathscr{I}_{\infty}^{\nearrow}(\mathbb{Z})$ as a dense discrete subsemigroup. If $I=S \backslash \mathscr{I}_{\infty}^{\nearrow}(\mathbb{Z}) \neq \varnothing$ then $I$ is an ideal of $S$.

Proof. Suppose that $I$ is not an ideal of $S$. Then at least one of the following conditions holds:

$$
\text { 1) } \left.I \cdot \mathscr{I}_{\infty}^{\nearrow}(\mathbb{Z}) \nsubseteq I, \quad \text { 2) } \mathscr{I}_{\infty}^{\nearrow}(\mathbb{Z}) \cdot I \nsubseteq I, \quad \text { or } \quad 3\right) I \cdot I \nsubseteq I .
$$

Since $\mathscr{I}_{\infty}^{\nearrow}(\mathbb{Z})$ is a dense discrete subspace of $S$, Theorem 3.5 .8 from 14 implies that $\mathscr{I}_{\infty}^{\nearrow}(\mathbb{Z})$ is an open subspace of $S$. Suppose there exist $\alpha \in \mathscr{I}_{\infty}^{\nearrow}(\mathbb{Z})$ and $\beta \in I$ such that $\beta \cdot \alpha=\gamma \notin I$. Since $\mathscr{I}_{\infty}^{\nearrow}(\mathbb{Z})$ is a dense open discrete subspace of $S$, the continuity of the semigroup operation in $S$ implies that there exists an open neighbourhood $U(\beta)$ of $\beta$ in $S$ such that $U(\beta) \cdot\{\alpha\}=\{\gamma\}$. Hence we have that $\left(U(\beta) \cap \mathscr{I}_{\infty}^{\nearrow}(\mathbb{Z})\right) \cdot\{\alpha\}=\{\gamma\}$ and the set $U(\beta) \cap \mathscr{I}_{\infty}^{\nearrow}(\mathbb{Z})$ is infinite. But by Proposition 2.5, the equation $\chi \cdot \alpha=\gamma$ has finitely many solutions in $\mathscr{I}_{\infty}^{\nearrow}(\mathbb{Z})$. This contradicts the assumption that $\beta \in S \backslash \mathscr{I}_{\infty}^{\nearrow}(\mathbb{Z})$. Therefore $\beta \cdot \alpha=\gamma \in I$ and hence $I \cdot \mathscr{I}_{\infty}^{\nearrow}(\mathbb{Z}) \subseteq I$. The proof of the inclusion $\mathscr{I}_{\infty}^{\nearrow}(\mathbb{Z}) \cdot I \subseteq I$ is similar.

Suppose there exist $\alpha, \beta \in I$ such that $\alpha \cdot \beta=\gamma \notin I$. Since $\mathscr{I}_{\infty}^{\nearrow}(\mathbb{Z})$ is a dense open discrete subspace of $S$, the continuity of the semigroup operation in $S$ implies that there exist open neighbourhoods $U(\alpha)$ and $U(\beta)$ of $\alpha$ and $\beta$ in $S$, respectively, such that $U(\alpha) \cdot U(\beta)=\{\gamma\}$. Hence we have that $\left(U(\beta) \cap \mathscr{I}_{\infty}^{\nearrow}(\mathbb{Z})\right) \cdot\left(U(\alpha) \cap \mathscr{I}_{\infty}^{\nearrow}(\mathbb{Z})\right)=\{\gamma\}$ and the sets $U(\beta) \cap \mathscr{I}_{\infty}^{\nearrow}(\mathbb{Z})$ and $U(\alpha) \cap \mathscr{I}_{\infty}^{\nearrow}(\mathbb{Z})$ are infinite. But by Proposition 2.5, the equations $\chi \cdot \beta=\gamma$ and $\alpha \cdot \kappa=\gamma$ have finitely many solutions in $\mathscr{I}_{\infty}^{\nearrow}(\mathbb{Z})$. This contradicts the assumption that $\alpha, \beta \in S \backslash \mathscr{I}_{\infty}^{\nearrow}(\mathbb{Z})$. Therefore $\alpha \cdot \beta=\gamma \in I$ and hence $I \cdot I \subseteq I$.

Proposition 4.7. Let $S$ be a Hausdorff topological semigroup which contains $\mathscr{I}_{\infty}^{\nearrow}(\mathbb{Z})$ as a dense discrete subsemigroup. Then for every $\gamma \in \mathscr{I}_{\infty}^{\nearrow}(\mathbb{Z})$ the set

$$
D_{\gamma}=\left\{(\chi, \varsigma) \in \mathscr{I}_{\infty}^{\nearrow}(\mathbb{Z}) \times \mathscr{I}_{\infty}^{\nearrow}(\mathbb{Z}) \mid \chi \cdot \varsigma=\gamma\right\}
$$

is a closed-and-open subset of $S \times S$.

Proof. Since $\mathscr{I}_{\infty}^{\nearrow}(\mathbb{Z})$ is a discrete subspace of $S$ we have that $D_{\gamma}$ is an open subset of $S \times S$.

Suppose that there exists $\gamma \in \mathscr{I}_{\infty}^{\nearrow}(\mathbb{Z})$ such that $D_{\gamma}$ is a non-closed subset of $S \times S$. Then there exists an accumulation point $(\alpha, \beta) \in S \times S$ of the set $D_{\gamma}$. The continuity of the semigroup operation in $S$ implies that $\alpha \cdot \beta=\gamma$. But $\mathscr{I}_{\infty}^{\nearrow}(\mathbb{Z}) \times \mathscr{I}_{\infty}^{\nearrow}(\mathbb{Z})$ is a discrete subspace of $S \times S$ and hence by Theorem 4.6, 
the points $\alpha$ and $\beta$ belong to the ideal $I=S \backslash \mathscr{I}_{\infty}^{\nearrow}(\mathbb{Z})$ and hence $\alpha \cdot \beta \in S \backslash \mathscr{I} \Phi_{\infty}^{\nearrow}(\mathbb{Z})$ cannot be equal to $\gamma$.

Theorem 4.8. If a Hausdorff topological semigroup $S$ contains $\mathscr{I}_{\infty}^{\nearrow}(\mathbb{Z})$ as a dense discrete subsemigroup then the square $S \times S$ cannot be pseudocompact.

The proof of Theorem 4.8 is similar to that of Theorem 5.1(3) of [6].

Recall that, a topological semigroup $S$ is called $\Gamma$-compact if for every $x \in S$ the closure of the set $\left\{x, x^{2}, x^{3}, \ldots\right\}$ is a compactum in $S$ (see [20]). We recall that the Stone-Cech compactification of a Tychonoff space $X$ is a compact Hausdorff space $\beta X$ containing $X$ as a dense subspace so that each continuous map $f: X \rightarrow Y$ to a compact Hausdorff space $Y$ extends to a continuous map $\bar{f}: \beta X \rightarrow Y$ [14].

Corollary 4.9. If a topological semigroup $S$ satisfies one of the following conditions: ( $i) S$ is compact; (ii) $S$ is $\Gamma$-compact; (iii) the square $S \times S$ is countably compact; (iv) $S$ is a countably compact topological inverse semigroup; or $(v)$ the square $S \times S$ is a Tychonoff pseudocompact space, then $S$ does not contain the semigroup $\mathscr{I}_{\infty}^{\nearrow}(\mathbb{Z})$ (and hence the semigroup $\mathscr{I}_{\infty}^{\rightarrow}(\mathbb{Z})$ ).

Proof. By Theorem 2 from [22] every compact topological semigroup is stable. But by Corollary 3.1 of [3] a stable semigroup cannot contain the bicyclic semigroup. Since by Remark 1.2, for any positive integer $n$ the semigroup $\mathscr{I}_{\infty}^{\nearrow}(\mathbb{Z})$ contains the semigroup $\mathscr{C}(n,+)$ which is isomorphic to the bicyclic semigroup we conclude that any compact topological semigroup cannot contain the semigroup $\mathscr{I}_{\infty}^{\nearrow}(\mathbb{Z})$.

Similarly by Proposition 5.3 of [20] no $\Gamma$-compact topological semigroup can contain the bicyclic semigroup. Also the proof of Theorem 10 from [5] implies that every topological semigroup $S$ with countably compact square $S \times S$ cannot contain the bicyclic semigroup and by Theorem 1 from [17] any countably compact topological inverse semigroup cannot contain the bicyclic semigroup, either. Next we apply Remark 1.2.

By Theorem 1.3 from [4] for any topological semigroup $S$ with the pseudocompact square $S \times S$ the semigroup operation $\mu: S \times S \rightarrow S$ extends to a continuous semigroup operation $\beta \mu: \beta S \times \beta S \rightarrow \beta S$, so $\mathrm{S}$ is a subsemigroup of the compact topological semigroup $\beta S$. Therefore if $S$ contains the bicyclic semigroup then $\beta S$ also contains the bicyclic semigroup which is a contradiction.

The proofs of the following three theorems are similar to Theorems 4.1, 4.6 and 4.8, respectively.

Theorem 4.10. Every Baire topology $\tau$ on $\mathscr{I}_{\infty}^{\rightarrow}(\mathbb{Z})$ such that $\left(\mathscr{I}_{\infty}^{\rightarrow}(\mathbb{Z}), \tau\right)$ is a Hausdorff semitopological semigroup is discrete.

Theorem 4.11. Let $S$ be a topological semigroup which contains $\mathscr{I}_{\infty}^{\rightarrow \rightarrow}(\mathbb{Z})$ as a dense discrete subsemigroup. If $I=S \backslash \mathscr{I}_{\infty}^{\rightarrow}(\mathbb{Z}) \neq \varnothing$ then $I$ is an ideal of $S$.

Theorem 4.12. If a Hausdorff topological semigroup $S$ contains $\mathscr{I}_{\infty}^{\rightarrow}(\mathbb{Z})$ as a dense discrete subsemigroup then the square $S \times S$ cannot be pseudocompact.

Remark 4.13. We observe that the topology $\tau_{W}^{\rightarrow \rightarrow}$ on the semigroup $\mathscr{I}_{\infty}^{\rightarrow}(\mathbb{Z})$ which is generated by the family

$$
\mathscr{B}_{W}^{q}(\alpha)=\left\{U_{\alpha}(F) \mid F \text { is a finite subset of } \operatorname{dom} \alpha\right\}
$$

where

$$
U_{\alpha}(F)=\left\{\beta \in \mathscr{I}_{\infty}^{\rightarrow}(\mathbb{Z}) \mid \operatorname{dom} \beta \subseteq \operatorname{dom} \alpha \text { and }(x) \beta=(x) \alpha \text { for all } x \in F\right\},
$$

is a non-discrete inverse semigroup topology. The proof of continuity of the semigroup operation and inversion in $\left(\mathscr{I}_{\infty}^{\rightarrow}(\mathbb{Z}), \tau_{W}^{\rightarrow}\right)$ is similar to the proof of Proposition 4.5 and obviously the topology $\tau_{W}^{\rightarrow}$ induces the topology $\tau_{W}$ on the subsemigroup $\mathscr{I}_{\infty}^{\nearrow}(\mathbb{Z})$.

The following example shows that there exists a non-discrete Tychonoff topology $\tau_{H}^{\rightarrow}$ on the semigroup $\mathscr{I}_{\infty}^{\rightarrow}(\mathbb{Z})$ such that $\left(\mathscr{I}_{\infty}^{\rightarrow}(\mathbb{Z}), \tau_{H}^{\rightarrow}\right)$ is a topological inverse semigroup, every $H$-class in $\mathscr{I}_{\infty}^{\stackrel{\rightarrow}{(}}(\mathbb{Z})$ is an open subset in $\left(\mathscr{I}_{\infty}^{\rightarrow}(\mathbb{Z}), \tau_{H}^{\rightarrow \rightarrow}\right)$ and the topology $\tau_{H}^{\rightarrow \rightarrow}$ is finer than the topology $\tau_{W}^{9}$. 
Example 4.14. We define a topology $\tau_{H}^{\rightarrow}$ on the semigroup $\mathscr{I}_{\infty}^{\rightarrow}(\mathbb{Z})$ as follows. For every $\alpha \in \mathscr{I}_{\infty}^{q \rightarrow}(\mathbb{Z})$ we define a family

$$
\mathscr{B}_{H}^{\rightarrow}(\alpha)=\left\{W_{\alpha}(F) \mid F \text { is a finite subset of } \operatorname{dom} \alpha\right\}
$$

where

$$
W_{\alpha}(F)=\left\{\beta \in \mathscr{I}_{\infty}^{\mapsto}(\mathbb{Z}) \mid \beta \mathscr{H} \alpha \text { and }(x) \beta=(x) \alpha \text { for all } x \in F\right\} .
$$

It is straightforward to verify that $\left\{\mathscr{B}_{H}^{\rightarrow}(\alpha)\right\}_{\alpha \in \mathscr{I}_{\infty}^{\rightarrow}(\mathbb{Z})}$ forms a basis for a topology $\tau_{H}^{\rightarrow \rightarrow}$ on the semigroup $\mathscr{I}_{\infty}^{\rightarrow \rightarrow}(\mathbb{Z})$.

Proposition 4.15. $\left(\mathscr{I}_{\infty}^{\rightarrow}(\mathbb{Z}), \tau_{H}^{\rightarrow}\right)$ is a Tychonoff topological inverse semigroup.

Proof. The proof of continuity of the semigroup operation and inversion in $\left(\mathscr{I}_{\infty}^{\rightarrow}(\mathbb{Z}), \tau_{H}^{\rightarrow \rightarrow}\right)$ is similar to the proof of Proposition 4.5. Also the definition of the topology $\tau_{H}^{9}$ implies that all $H$-classes are open subsets in $\left(\mathscr{I}_{\infty}^{\rightarrow}(\mathbb{Z}), \tau_{H}^{\rightarrow \rightarrow}\right)$ and the group of units $H(\mathbb{I})$ of the semigroup $\mathscr{I}_{\infty}^{\rightarrow}(\mathbb{Z})$ with the induced topology from $\left(\mathscr{I}_{\infty}^{\rightarrow}(\mathbb{Z}), \tau_{H}^{\rightarrow}\right)$ is a non-discrete topological group, and hence by Theorem II.8.4 from [19] the topological subspace $H(\mathbb{I})$ is Tychonoff. Hence since every $H$-class in topological inverse semigroup $S$ is a closed subset in $S$ (see [13]), Proposition 3.1, Corollary 3.4 and Theorem 2.20 of [9] imply that the topological space $\left(\mathscr{I}_{\infty}^{\rightarrow}(\mathbb{Z}), \tau_{H}^{\rightarrow}\right)$ is homeomorphic to topological sum of a countable many of topological spaces $H(\mathbb{I})$, and hence $\left(\mathscr{I}_{\infty}^{\rightarrow}(\mathbb{Z}), \tau_{H}^{\rightarrow}\right)$ is a Tychonoff space.

We observe that the topology $\tau_{H}^{\rightarrow}$ on the semigroup $\mathscr{I}_{\infty}^{\rightarrow}(\mathbb{Z})$ induces the discrete topology on its subsemigroup $\mathscr{I}_{\infty}^{\nearrow}(\mathbb{Z})$.

Recall [11] that a Bohr compactification of a topological semigroup $S$ is a pair $(\beta, B(S))$ such that $B(S)$ is a compact topological semigroup, $\beta: S \rightarrow B(S)$ is a continuous homomorphism, and if $g: S \rightarrow T$ is a continuous homomorphism of $S$ into a compact semigroup $T$, then there exists a unique continuous homomorphism $f: B(S) \rightarrow T$ such that the diagram

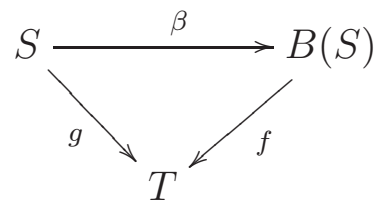

commutes. Then Theorems [2.9 and 3.7, and Proposition 2 from [2] imply the following:

Corollary 4.16. The Bohr compactification of the discrete semigroup $\mathscr{I}_{\infty}^{\nearrow}(\mathbb{Z})\left(\mathscr{I}_{\infty}^{q}(\mathbb{Z})\right)$ is topologically isomorphic to the Bohr compactification of discrete group $\mathbb{Z}(+) \times \mathbb{Z}(+)$.

\section{ACKNOWLEDGEMENTS}

This research was supported by the Slovenian Research Agency grants P1-0292-0101, J1-4144-0101 and BI-UA/11-12-001. The authors are grateful to the referee for useful comments and suggestions.

\section{REFERENCES}

[1] O. Andersen, Ein Bericht über die Struktur abstrakter Halbgruppen, PhD Thesis, Hamburg, 1952.

[2] L. W. Anderson, R. P. Hunter, On the compactification of certain semigroups, Contrib. Extens. Theory Topol. Struct. Proc. Sympos. Berlin 1967. VEB Deutscher Verlag der Wissenschaften, Berlin, 1969, 21 - 27.

[3] L. W. Anderson, R. P. Hunter and R. J. Koch, Some results on stability in semigroups. Trans. Amer. Math. Soc. 117 (1965), $521-529$.

[4] T. Banakh and S. Dimitrova, Openly factorizable spaces and compact extensions of topological semigroups, Comment. Math. Univ. Carol. 51:1 (2010), 113-131.

[5] T. Banakh, S. Dimitrova and O. Gutik, The Rees-Suschkiewitsch Theorem for simple topological semigroups, Mat. Stud. 31:2 (2009), 211-218.

[6] T. Banakh, S. Dimitrova and O. Gutik, Embedding the bicyclic semigroup into countably compact topological semigroups, Topology Appl. 157:18 (2010), 2803-2814.

[7] M. O. Bertman and T. T. West, Conditionally compact bicyclic semitopological semigroups, Proc. Roy. Irish Acad. A76:21-23 (1976), 219-226.

[8] J. H. Carruth, J. A. Hildebrant and R. J. Koch, The Theory of Topological Semigroups, Vol. I, Marcel Dekker, Inc., New York and Basel, 1983; Vol. II, Marcel Dekker, Inc., New York and Basel, 1986. 
[9] A. H. Clifford and G. B. Preston, The Algebraic Theory of Semigroups, Vol. I., Amer. Math. Soc. Surveys 7, Providence, R.I., 1961; Vol. II., Amer. Math. Soc. Surveys 7, Providence, R.I., 1967.

[10] W. W. Comfort, Topological groups, Handbook of set-theoretic topology, Kunen K., Vaughan J. (eds.) Elsevier, 1984, pp. 1143-1263.

[11] K. DeLeeuw, and I. Glicksberg, Almost-periodic functions on semigroups, Acta Math. 105 (1961), 99-140.

[12] J. D. Dixon and B. Mortimer, Permutation Groups, Graduate Texts in Math. 163, Springer, New York, 1996.

[13] C. Eberhart and J. Selden, On the closure of the bicyclic semigroup, Trans. Amer. Math. Soc. 144 (1969), $115-126$.

[14] R. Engelking, General Topology, 2nd ed., Heldermann, Berlin, 1989.

[15] O. V. Gutik and K. P. Pavlyk, On topological semigroups of matrix units, Semigroup Forum 71:3 (2005), 389-400.

[16] O. Gutik, K. Pavlyk and A. Reiter, Topological semigroups of matrix units and countably compact Brandt $\lambda^{0}$ extensions, Mat. Stud. 32:2 (2009), 115-131.

[17] O. Gutik and D. Repovš, On countably compact 0-simple topological inverse semigroups, Semigroup Forum 75:2 (2007), 464-469.

[18] O. Gutik and D. Repovš, Topological monoids of monotone, injective partial selfmaps of $\mathbb{N}$ having cofinite domain and image, Stud. Sci. Math. Hungar. 48:3 (2011), 342-353.

[19] E. Hewitt and K. A. Roos, Abstract Harmonic Analysis, Vol. 1, Springer, Berlin, 1963.

[20] J. A. Hildebrant and R. J. Koch, Swelling actions of $\Gamma$-compact semigroups, Semigroup Forum 33 (1986), 65-85.

[21] J. M. Howie, Fundamentals of Semigroup Theory, London Math. Monographs, New Ser. 12, Clarendon Press, Oxford, 1995.

[22] R. J. Koch and A. D. Wallace, Stability in semigroups, Duke Math. J. 24 (1957), 193-196.

[23] A. A. Markov, On free topological groups, Izvestia Akad. Nauk SSSR 9 (1945), 3- 64 (in Russian); English version in: Transl. Amer. Math. Soc. (1) 8 (1962), 195- 272.

[24] W. D. Munn, Uniform semilattices and bisimple inverse semigroups, Quart. J. Math. 17:1 (1966), 151-159.

[25] A. Yu. Ol'shanskiy, Remark on countable non-topologized groups, Vestnik Moscow Univ. Ser. Mech. Math. 39 (1980), 1034 (in Russian).

[26] M. Petrich, Inverse Semigroups, John Wiley \& Sons, New York, 1984.

[27] L. S. Pontryagin, Topological Groups, Gordon \& Breach, New York ets, 1966.

[28] A. D. Taimanov, The topologization of commutative semigroups, Mat. Zametki 17:5 (1975), 745 - 748 (in Russian).

[29] V. V. Wagner, Generalized groups, Dokl. Akad. Nauk SSSR 84 (1952), 1119-1122 (in Russian).

Department of Mechanics and Mathematics, Ivan Franko Lviv National University, Universytetska

1, LVIV, 79000, UKRAINE

E-mail address: o_gutik@franko.lviv.ua, ovgutik@yahoo.com

Faculty of Education, and Faculty of Mathematics and Physics, University of Ljubljana, P. O. B. 2964, Ljubluana, 1001, Slovenia

E-mail address: dusan.repovs@guest.arnes.si 